\title{
Urinary Retention: Catheter Drainage Bag or Catheter Valve?
}

\author{
Gurnam Virdi David Hendry \\ Department of Urology, Queen Elizabeth University Hospital, Glasgow, UK
}

\section{Key Words}

Urinary retention $\cdot$ Voiding $\cdot$ Catheter

\begin{abstract}
The management of patients with intractable urinary incontinence presents as a challenging priority in the ageing population. To preserve the antibacterial mechanisms of the bladder, a urine collection that enables the bladder to fill and empty regularly and completely, should be used. This mimics the action of the healthy bladder. We compared the success rates of two widely used urinary-collection systems (catheter with drainage bag or a catheter valve) at our institution for those patients undergoing a trial of void.
\end{abstract}

Copyright $\odot 2015$ S. Karger AG, Basel

\section{Introduction}

In this era when the quality of life for so many patients has been restored by various medical advances, there still remains a need for a low-risk, inexpensive, and patient-ideal urine collection system, especially for those undergoing a trial of voiding (TOV). One effective method of relieving the patient from the inability to void urine is by catheterising them i.e. TOV for a short period of time. Trial of voiding assesses a patient's ability to urinate after the removal of an indwelling catheter with an aim to retain/restore bladder capacity.

\section{KARGER}

Fax +41613061234

E-Mail karger@karger.ch

www.karger.com
(C) 2015 S. Karger AG, Basel

1015-9770/14/0091-0028\$38.00/0

Accessible online at:

www.karger.com/cur
The two most common voiding systems used in our institution are a catheter with drainage bag and a catheter valve. Both have their advantages and disadvantages. At our institution, our observations noted that patients who underwent a TOV with a catheter valve had fewer infection rates as opposed with those undergoing a TOV with a catheter attached to a leg bag. This was the basis of this audit.

\section{Aims}

The aim of this study was to audit the success of the two type of catheter collection systems used in the management of all patients with the inability to void urine at our institution.

\section{Objectives}

Our three main objectives were to: establish the indication for a catheter and TOV; establish whether the catheter used was either draining freely into a leg bag or had a catheter valve; and compare success rates of either catheter types above and provide recommendations.

\section{Methods}

A proforma based on our objectives was created to ensure consistency in our data gathering. All data was gathered from the 'Integrated Care Pathway Trial of Voiding' documentation in the

Gurnam Virdi

Department of Urology, Queen Elizabeth University Hospital 1345 Govan Road, G51 4TF

Glasgow (UK)

E-Mailgv1@doctors.org.uk 
Table 1. Shows the success rate of patients undergoing a TOV with either a catheter valve or leg bag

\begin{tabular}{lll}
\hline & Leg bag & Flip-flo catheter valve \\
\hline Successful & $19(63 \%)$ & $5(83 \%)$ \\
Non-successful & $11(37 \%)$ & $1(17 \%)$ \\
\hline
\end{tabular}

patient medical notes during the study period (from beginning August 2012 till end September 2012 with 8 weeks duration). Inclusion criteria: both men and women of all ages that underwent a TOV were included.

The Integrated Care Pathway documentation is a description of the patient's anticipated care needs that should be addressed by the multidisciplinary team. Within this pathway document, bladder scan reports and hourly fluid charts allowed the post-voidal residual volumes to be determined. We could then determine whether the TOV was successful or not. Additional information about the patient's management such as the indication for TOV and the urine collection system used was obtained reviewing the patient's notes. On completion, all the data were analysed and the results tabulated so recommendations could be made.

\section{Results}

A total of 36 patients were identified as being suitable for undergoing a TOV. This group of patients included 30 males and 6 females, with the age ranging from 38 to 90 years old. Prior to TOV, all patients had:

- Ensured bowels were active

- Catheter specimen of urine was obtained (if positive then patients were given antibiotics, if negative patients underwent a TOV)

- Voiding instructions were given

- Patient information leaflet given (pelvic floor exercises and fluid balance)

All catheters were removed with informed consent and it was it was noted if the TOV was successful or unsuccessful.

Of the total number of patients who had a catheter with leg bag (30), $37 \%$ of them presented with a urinary tract infection (UTI) and the TOV had to be stopped so the patients could undergo a course of antibiotics for one week before they were re-trialled. In the group of patients who underwent a TOV with a catheter valve only one (17\%) presented with a UTI (table 1).

Twenty-eight (78\%) of the total 36 patients were given an alpha-blocker (Tamsulosin) during their period of catheterisation prior to the TOV. It was not documented why the remaining 8 patients did not receive this.

\section{Discussion}

In the United Kingdom, urinary retention is a commonly experienced problem among many elderly patients in hospitals. This condition has implications for patient well-being, length of stay and for the ability of the patient to return to independent living. There are several problems that can occur if urinary retention is not treated such as: recurrent or chronic urinary tract infection, overflow urinary tract incontinence, inability of the bladder to contract, and damage to the upper urinary tract.

Catheter valves have been around for many years and first introduced into the UK in 1986. They are a popular alternative to the more customary urine drainage bag and they simply fit into the end of a catheter either urethral or suprapubic.

The traditional method of allowing urine to drain freely into a bag can cause problems for patients - the bag can become heavy and uncomfortable. This can cause the patient to feel embarrassed and even depressed. This method can also be detrimental to the bladder and lead to loss of normal bladder function due to the fact a catheterised bladder is always empty and drains continuously. In addition, infection is a problem with drainage bags.

Patients prefer catheter valves as opposed to a leg bag as it is more discreet and comfortable. In one clinical study, $72 \%$ of patient's preferred a catheter valve to conventional drainage bags [2]. Many patients did not like the sensation of urine "sloshing about" when using the drainage bag. However, some patients using the catheter valve did complain of nocturnal frequency therefore the authors suggested that a combination of a valve during the day and an overnight bag may be ideal. In another study, $92 \%$ of patients using a catheter valve reported being 'happy or satisfied' compared to $35 \%$ using a drainage bag [3]. Also in this study, the patients who used a drainage bag felt their level of activity was impaired. In addition, the catheter valve caused less morbidity because it is constructed to allow one-way flow and this was not associated with an increase in the incidence of urinary tract infections.

From this audit, it can be seen that the success rate is much greater $(83 \%)$ if undergoing a TOV with a catheter valve as opposed to only a success rate of $63 \%$ with a catheter leg bag. This supports the growing opinion and our observations that a catheter valve is more successful than a catheter leg bag when patients present with retention of urine. The occurrence of an infection is much 
greater in the leg bag group with $43 \%$ of patients ending up with a UTI as opposed to only one (17\%) patient in group of patients with a catheter valve.

These small devices (catheter valves) have another huge advantage which is that they offer the potential for maintenance of bladder function, capacity and tone by allowing the filling and emptying of the bladder, mimicking normal bladder function. In addition, valves reduce trauma to the bladder by allowing the bladder wall to be lifted away from the catheter. Research shows that using a catheter valve with a four-hourly release is associated with reduced catheter bloackage [1]. However, catheter valves are unsuitable for some patients. These include people with severe cognitive impairment, uncontrolled detrusor overactivity, high pressure chronic retention, ureteric reflux or obstructive renal impairment/failure. Dexterity can also be a problem though it can be argued that there is little difference between opening a catheter valve and opening a drainage bag tap.

\section{Recommendations}

This audit, however, does not come without any limitations, the biggest being the fact that this audit was not a randomised control trial. In addition, the numbers are relatively small therefore a much larger national audit of this nature would be required if any recommendations were to be put in place. A huge advantage of the catheter valve is that it will lower the cost burden on the NHS as they are greatly cheaper than catheterising with leg bags.

Our recommendation is that patients who present with urinary retention and are suitable for a TOV should be allowed to use catheter valves, unless contraindicated. From this audit, it is clearly evident that those undergoing a TOV with a catheter valve have less, if any, infection rates than those with a catheter leg bag.

\section{Conclusion}

Essentially, the bladder is like a muscle and it will eventually waste away if not used regularly. It has been demonstrated that after only 6 months on free-flow drainage using a leg bag, the bladder shrinks and ceases to function properly [4]. However, the use of a catheter valve has the potential to maintain bladder function, capacity and tone. From the perspective of the patient and the healthcare members, the regaining of bladder continence is a major achievement. By using a catheter valve, patients can enjoy greater personal freedom, emptying their bladders when it is convenient to them.

\section{References}

1 Sabbuba N, Stickler DJ, Long MJ, Dong Z, Short TD, Feneley RJ: Does the valve regulated release of urine from the bladder decrease encrustation and blockage of indwelling catheters by crystalline proteus mirabilis biofilms? J Urol 2005;173:262-266.
2 German K, Rowley P, Stone D, Kumar U, Blackford HN: A randomized cross-over study comparing the use of a catheter valve and a leg-bag in urethrally catheterized male patients. Br J Urol 1997;79:96-98.
3 Wilson C, Sandhu SS, Kaisary AV: A prospective randomized study comparing a catheter-valve with a standard drainage system. Br J Urol 1997;80:915-917.

4 Kristiansen P, Pompeius R, Wadström LB: Long-term urethral catheter drainage and bladder capacity. Neurourol Urodyn 1983;2: 135-143. 